\title{
Peak Detection Using Wavelet Transform
}

\author{
Omar Daoud ${ }^{1}$, Qadri Hamarsheh ${ }^{2}$, and Saleh Saraireh ${ }^{1}$ \\ ${ }^{1}$ Department of Communications and Electronics Engineering, Philadelphia University, \\ Amman, Jordan \\ ${ }^{2}$ Department of Computer Engineering, Philadelphia University, Amman, Jordan
}

\begin{abstract}
A new work based-wavelet transform is designed to overcome one of the main drawbacks that found in the present new technologies. Orthogonal Frequency Division Multiplexing (OFDM)is proposed in the literature to enhance the multimedia resolution. However, the high peak power (PAPR) values will obstruct such achievements. Therefore, a new proposition is found in this work, making use of the wavelet transforms methods, and it is divided into three main stages; de-noising stage, thresholding stage and then the replacement stage.
\end{abstract}

In order to check the system stages validity; a mathematical model has been built and its checked after using a MATLAB simulation. A simulated bit error rate (BER) achievement will be compared with our previously published work, where an enhancement from $8 \times 10^{-1}$ to be $5 \times 10^{-1}$ is achieved. Moreover, these results will be compared to the work found in the literature, where we have accomplished around $27 \%$ PAPR extra reduction.

As a result, the BER performance has been improved for the same bandwidth occupancy. Moreover and due to the de-noise stage, the verification rate has been improved to reach $81 \%$. This is in addition to the noise immunity enhancement.

\section{KEYWORDS}

MIMO-OFDM, PAPR, wavelet- eigen vector, de-noising

\section{INTRODUCTION}

In order to enhance the multimedia performance, many generations have been evolved during the last decade. This will complicate the modern wireless technologies, where a devastating massive data that the users' can deal with; create, transmit and/or manage. This is a main reason behind turning the researcher's interest toward new solutions to ease such complexities. Thus, a combination between two solid technologies has been found in the literature in order to improve the system's reliability for either the downlink or the uplink transmissions and achieve a competitive data rates to be over $100 \mathrm{Mbps}$ and $30 \mathrm{Mbps}$, respectively; namely Orthogonal Frequency Division Multiplex (OFDM) and Multiple-Input Multiple-Output (MIMO) technology. Therefore, either wired or wireless multimedia applications growth will be controlled. This is in addition to produce a high capacity advanced wireless techniques.

Speaker verifications topic is consider as one of the challenging issues these days for the present wireless technologies. Here, the information properties have the researcher's concern and it is an active topic for more than several years and for a variety of applications and revealed as a difficult task to be considered [1]. The speaker verifications complexity came from the needed amount of speech details to verify, estimate, and decide either the acceptance or the rejection

DOI : $10.5121 /$ ijcnc. 2014.6410 
levels [1-4]. MIMO-OFDM system is an example about the developments found in the literature to meet the improvements requirements such as reliability and coverage.

An efficient use of the limited bandwidth has been fulfilled by imposing the OFDM technique, where the multicarrier/ parallel modulation is proposed for the channel effect remedy; frequency selective fading, multipath noise, spectral efficiency in addition to the implementation easiness. OFDM is easily implemented using the Fast Fourier Transforms (FFT) and its inverse, where they used to multiplex signals with orthogonal carriers together. Furthermore, it combat the channel effect; the inter-symbol interference (ISI); using the added cyclic prefixes (CP) before transmitting. Therefore, the transmitted signal will be longer than the coherence time of the channel for the cost of reducing the channel spectral containment [4]. One of the main challenges that is found in the OFDM is the high peak-to-average power ratio (PAPR), it is a result of the coherence addition of the orthogonal subcarriers in IFFT in the transmitting stage; this will be considered as a major drawback especially in the use of the nonlinear devices. The large envelope fluctuation will need a large dynamic range for such devices and then it will limit the overall system efficiency and will operate at lower average power. [5-10] represents various techniques to reduce the effect of the PAPR, where they are classified into two main categories; distortion and distortion-less method. As a results, [11-22] depicts the cost of such techniques where the this effect is reduced under the cost of increasing the receiver complexity in addition to the irreducible BER. Therefore, the overall system throughput is reduced. It is clearly defined that the envelope fluctuation could be defined as

$$
\mathrm{PAPR}=\frac{\left|\frac{1}{\sqrt{N}} \sum_{n=0}^{N-1} X_{n} e^{j 2 \pi \sigma_{0} n t}\right|^{2}}{\frac{1}{N T} \int_{0}^{N T}\left|\frac{1}{\sqrt{N}} \sum_{n=0}^{N-1} X_{n} e^{j 2 \pi \pi_{0} n t}\right|^{2} d t}
$$

Here, the nominator defines the OFDM symbol peak power and the denominator is the average power. The symbol duration is $T$, the $n$-th sub carrier is $X_{\mathrm{n}}$ and $f_{0}$ is the nominal subcarrier frequency spacing.

Our previously published work in [9, 23-24], shows a competitive technique that overcomes those complexity and based on a mathematical model that conclude the average power is equal to the number of modulated OFDM subcarriers. Thus starting from the average power definition as

$$
P_{\text {avg }}=\Sigma\left\{\left|x_{i}(t)\right|^{2}\right\}=\frac{1}{T} \int_{0}^{T}|x(t)|^{2} d t=\frac{1}{T} \iint_{0}^{T}\left|\sum_{v=0}^{N-1} c_{v} e^{j 2 \pi} \frac{v}{N T} t_{v}\right|^{2} d t
$$

Here $c_{v}$ is the magnitude of the modulated data and for simplicity let us use the simplest modulation technique; BPSK with $|c \boldsymbol{v}|=1$. (2) could be reduced to leads to

$$
P_{\mathrm{avg}}=N \frac{1}{T} \int_{0}^{T} c_{v}^{2} d t=N
$$

Then a direct relationship between the average power and the total number the IFFT points, $N$, is achieved. This means that the PAPR reduction is accomplished by reducing the average power of the OFDM symbol. As a result, the computational complexity will be overcome over the one that has been found in the literature.

In this work and as will be shown in the next section, a new work will be proposed based on the wavelet transform namely OFDM based on the wavelet transform (WT) method; (DWT-OFDM). This work performance will be checked based on either the PAPR reduction or the BER 
reduction. Making use of the definition that is found in [22] which is based on Chernoff Union bound; the effective SINR must accomplish the following relationship as

$$
\mathrm{BER}(\mathrm{SINR})=B E R_{W G N}\left(-\lambda \ln \left(\frac{1}{N Q} \sum_{q=1}^{Q} \sum_{n=1}^{N} \exp \left(\frac{-S I N R_{q}}{\lambda}\right)\right)\right)
$$

Where the SINR is defined by the ratio of the expected values between the useful information and the interference ones. Then, the BER could be considered as the wrong detection or the errors that resulted from a noisy channel and it is the relationship between the presence of eror probabilities and the signal to noise ratio. In (4), $n$ is the $n$-th subcarrier and has the values between 1 to $N, \lambda$ is a unique parameter based on the system level simulation, $Q$ is the set of symbols that will be transmitted through certain number of antennas.

The rest of paper is organized as follows; the introduced structure of the MIMO-OFDM system models is defined in Section 2, the numerical and simulation results are presented in Section 3, while the last section summarizes the conclusion.

\section{The MIMO-OFDM System Descrition}

In this subsection a new algorithm has been proposed to allocate the high peaks that found in an OFDM signal. As shown in Figure 1, the flowchart explains the following procedure to attain the target from the proposed algorithm. Thus, the proposed algorithm starts with scan the produced OFDM signal after the Fast Fourier Transform process (FFT)

This signal will be processed as follows:

1) The preprocess stage:

- $\quad$ Remove the noise from a signal using wavelet technology.

- $\quad$ Perform P-level Haar wavelet decomposition of a signal ( $\mathrm{P}=8)$; it is decomposed into two subsignals; to running average or trend; and to running difference or fluctuation.

- $\quad$ Construct the approximations, $C A_{P}$ and the details $C D_{P}$.

\section{2) Using Zero Crossing mechanism allocate the peaks and valleys matrices}

For all details coefficients where the peaks and valleys are lie because of the high pass filters used in wavelet transform.

3) The entropy for both of the preprocessed OFDM signal and the decomposed PLevels has been calculated using Shannon method.

Case Studies based on the decomposition acceptance:

1. Case Study 1: Detect true and false local extremes points using all details coefficients.

2. Case Study 2: Detect true and false local extremes points using details coefficients ( CD1, DD2).

3. Case Study 3: Detect true and false local extremes points using all details coefficients except ( $\mathrm{CD} 3, \mathrm{CD} 7$ and $\mathrm{CD} 8$ ).

4. Case Study 4: Detect true and false local extremes points for each packet details coefficients separately. 
International Journal of Computer Networks \& Communications (IJCNC) Vol.6, No.4, July 2014

4) Use moving average (MA) filter. This filter is used to find high peaks for the founded matrix's points with their neighbors surrounding them in original OFDM signal. Then save the result into corresponding points of the original signal.

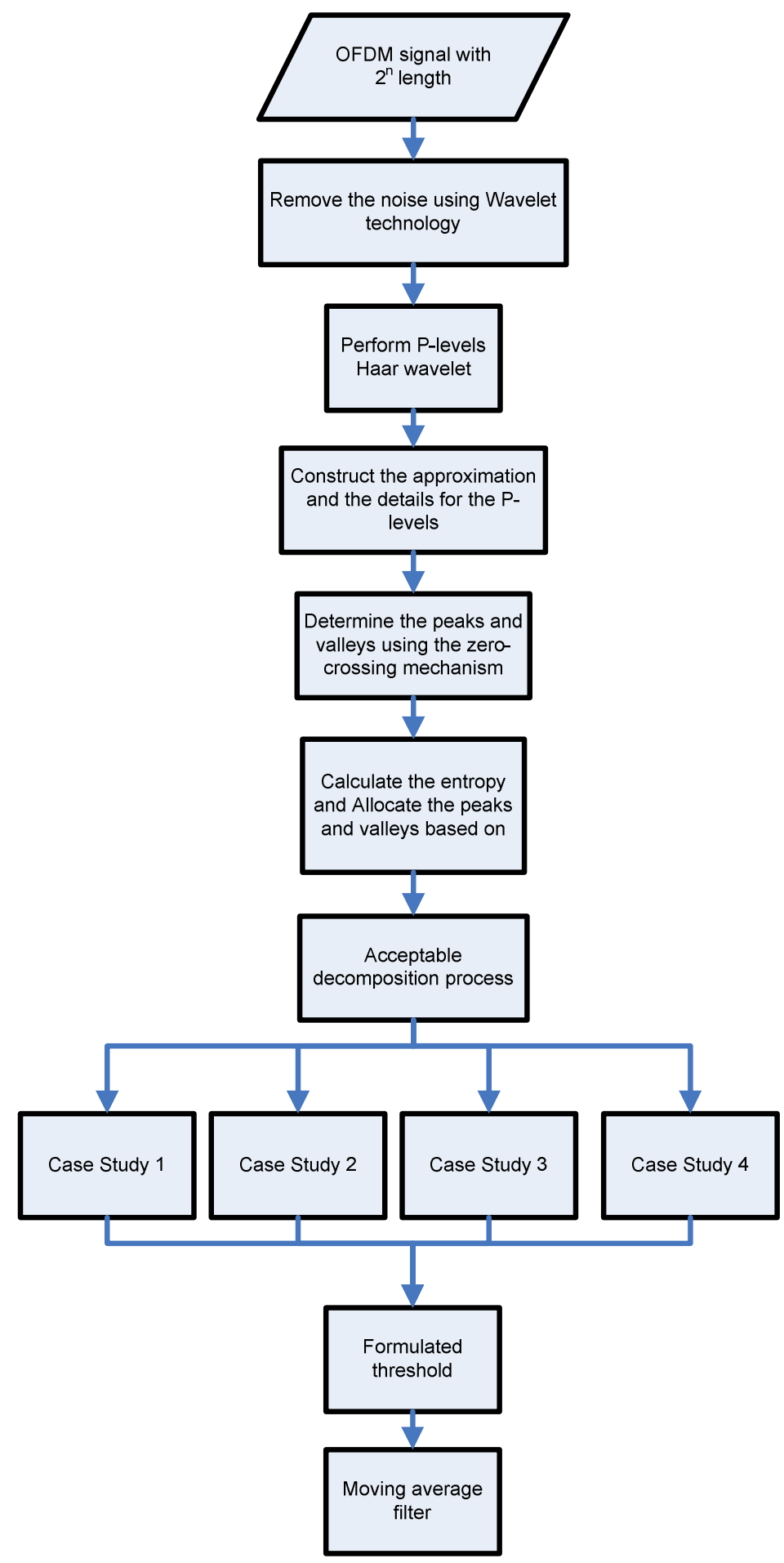

Figure 1. Flowchart of the proposed algorithm

In this section, a new technique has been proposed to allocate peaks in the OFDM signal based on the entropy wavelet packets. After dividing the used signal into eight levels using the wavelet 
packets, the entropy has been divided into the range of 0.68262 to 74.2432 . Based onto the Plevel summation, 4 case studies have been explained and found the best peaks allocation criterion; case study 4 . In this case study, a total of 414 peaks have been found with an error ratio of around $4 \%$. Furthermore, a predetermined threshold has been used to be compatible with various wireless systems specifications, and then these peaks have been replaced using the moving average filter to avoid the transmission with high peaks.

\section{Simulation ReSUlts AND DiscuSSION}

A MATLAB simulation has been performed to check the proposed system's performance and to be compared with both of the conventional techniques and our previously published work [9, 2224] under equivalent conditions. The simulation has been limited to the following factors:

- The input data is divided into two main categories:

- A practical data that is recorded data with $4 \mathrm{kHz}$ bandwidth sampled using the minimum sampling rate over a duration of $2 \mathrm{~s}$ time. It will be converted into binary bits using an ordinary A/D convertor with the three main steps shifting, normalizing and then quantizing.

- A theoretical data that is randomly generated,

- 256 points FFT size,

- Coding block is the convolutional encoder with $1 / 2$ coding rate, and

- QPSK modulation techniques.

Based on the simulation results it is clearly shown that the dense modulation technique gives better result than the low one. Moreover, it is clearly shown that the proposed work has better performance than either some techniques found in the literature such as clipping technique and partial transmit sequence (PTS) or our previously published work found in [23].

It is clearly found that the proposed work enhances system performance as shown in Figures 2; the BER has been improved to be $5.5 \times 10^{-1}$ while it was $8 \times 10^{-1}$. 


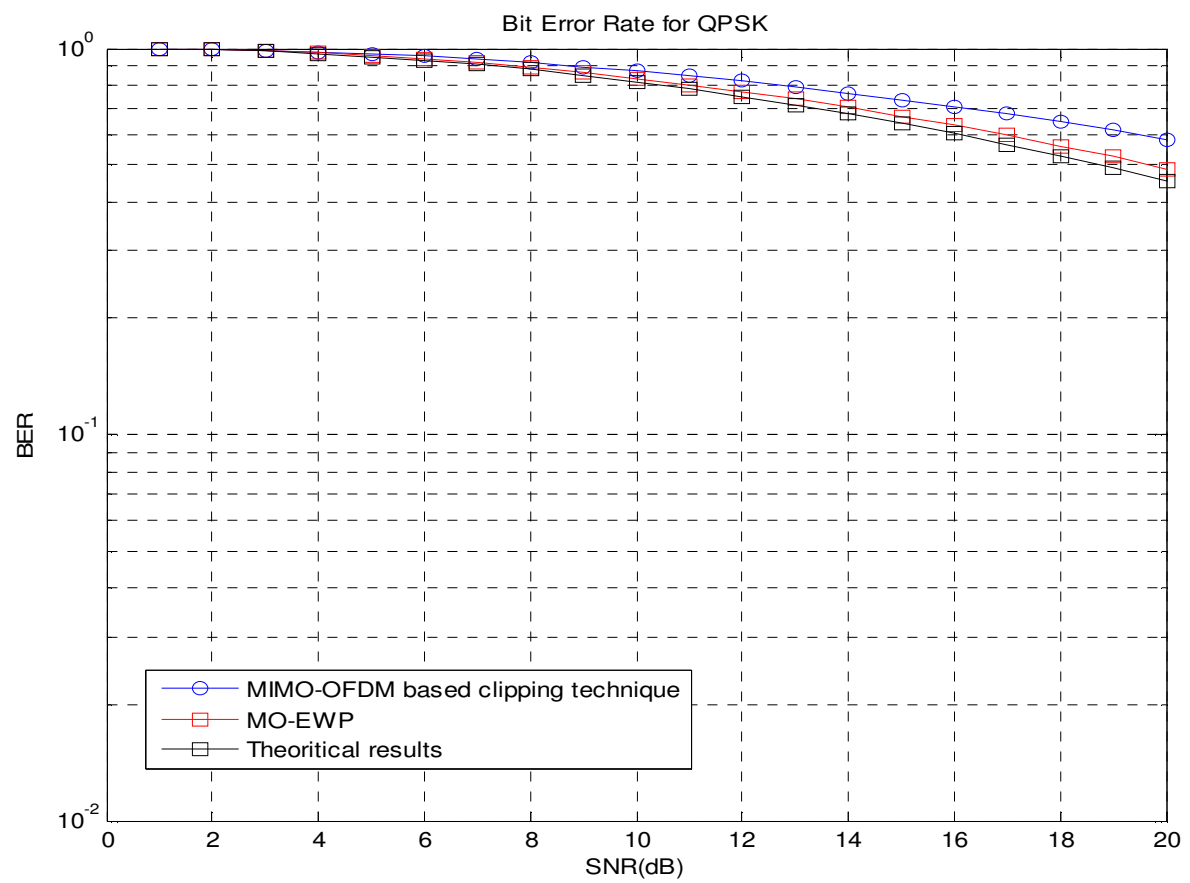

Figure 2. BER results based QPSK modulation Technique

\section{Conclusions}

A new proposition has been made in this paper to allocate the peaks and overcome the effect of high PAPR problem in MIMO-OFDM systems. It is based on both entropy of the wavelet coefficients and the moving average filters. The overall MIMO-OFDM system's performance is checked using a MATLAB simulation in addition to check the validity of the analytical derivation. It shows a promising performance improvement based on the found BER, where they have been exposed clearly in the previous section from $8 \times 10^{-1}$ to be $5 \times 10^{-1}$ at the same system threshold as an example. Moreover, it shows that $80 \%$ extra reduction has been achieved over the used techniques in the literature such as clipping and PTS techniques. Furthermore, an extra 15\% improvement has been achieved over our previously published work in [23].

\section{REFERENCES}

[1] Van Zelst A., "Space Division Multiplexing Algorithms ",10th Mediterranean Electrotechnical Conference, vol 3, 2000.

[2] Giangaspero L., et al., "Co-Channel Interference Cancellation based on MIMO OFDM Systems," IEEE Wireless Communications magazine, pp. 817, December, 2002.

[3] Bender P., et al.,"CDMA/HDR: a bandwidth efficient high speed wireless data service for nomadic users,” IEEE Communication Magazine, vol. 38, pp. 70-77, July, 2000.

[4] Lu B., et al., "LDPC-based space-time coded OFDM systems over correlated fading channels," IEEE Transaction in Communications, vol. 50, pp.74-88, Jan. 2002

[5] Brink S. T., et al., "Design of low-density parity check codes for multi-antennas modulation and detection," IEEE Transaction in Communications, vol. 52, no. 4, PP. 670- 678, April 2004.

[6] Reimers U., "Digital Video Broadcasting," IEEE Communication Magazine, vol. 36, no. 10, pp. 10410, Jun. 1998. 
International Journal of Computer Networks \& Communications (IJCNC) Vol.6, No.4, July 2014

[7] Nee V. and Prasad R., “OFDM wireless multimedia communications”, Artech House Boston London 2000.

[8] Jiang T. and Zhu G., "OFDM Peak-to-Average power Ratio reduction by Complement Block Coding Scheme and Its Modified Version,” Vehicular Technology Conference, vol. 1, pp. 448 - 512004.

[9] Al-Akaidi M., Daoud O. and Gow J., "MIMO-OFDM-based DVB-H systems: A Hardware design for a PAPR reducing technique", IEEE Transaction on Consumer Electronics, vol. 52, issue 4, pp. 12011206, Nov 2006.

[10] Heung-Gyoon and Kyoung-Jae Youn, "A New PAPR Reduction Scheme: SPW (Subblock Phase Weighting)" IEEE Transaction on Consumer Electronics, vol.48, No.1, pp.8 1-89, February 2002.

[11] European Telecommunication Standards Institute (ETSI), "Digital Video Broadcasting; Second generation framing structure, channel coding and modulation systems for Broadcasting, Interactive Services, News Gathering and other broadband satellite applications”, TR 102 376, V 1.1.1 2005.

[12] Digital Video Broadcasting Group, “DVB-T2 Call for Technologies”, SB1644 r1, April 2007.

[13] Mansour M., and Shanbhag N., "A Novel Design Methodology for High-Performance Pro $\neg$ grammable Decoder Cores for AA-LDPC codes," IEEE Workshop on Signal Processing Systems (SiPS), Korea, 2003.

[14] Hocevar D. "LDPC Code Construction with Flexible Hard-ware Implementation," IEEE International Conference on Communications, pp. 2708 -2712, 2003.

[15] Chen Y. and Hocevar D., "An FPGA and ASIC Implemen $\neg$ tation of Rate 1/2 8088-b Irregular Low Density Parity Check Decoder", Global Telecommunications Conference, vol. 1, pp. 113-117, 2003.

[16] Saltzberg B. ,"Comparison of Single-Carrier and Multitone Digital Modulation for ADSL Applications,” IEEE Communication Magazine, vol. 36, no. 11, pp. 114-21, Nov. 1998.

[17] Juntti M., et al., "MIMO MC-CDMA Communications for Future Cellular Systems", IEEE Communication Magazine. Feb, pp. 118-124, 2005.

[18] Ning L., et al., "An Exhaustive Entropy Based SLM Method for PAPR Reduction of OFDM Systems", 6th (ICST) IEEE conference on Communications and Networking in China, pp. 118-122, 2011.

[19]Wunder G., "A theoretical framework for peak-to-average power control problem in OFDM transmission”, $\mathrm{PhD}$ thesis, technical University Berlin, 2004.

[20]Han S. and Lee J., "An overview of Peak-to-Average Power Ratio reduction techniques for Multicarrier Transmission”, IEEE Wireless Communications, pp. 56-65, Apr, 2005.

[21]Tarokh V. and Jafarkhani H., "On the Computation and Reduction of the Peak-to- Average Power Ratio in Multicarrier Communications," IEEE Transaction Communications, vol. 48, no.1, pp.37-44, 2000 .

[22]Ning L., et al., "A Novel SLM Method for PAPR Reduaction of OFDM System”, 75th IEEE Vehicular Technology Conference (VTC Spring), pp. 1-5, 2012.

[23]Al-Akaidi M., Daoud O., and Linfoot S., "A new Turbo Coding Approach to reduce the Peak-toAverage Power Ratio of a Multi-Antenna-OFDM", International Journal of Mobile Communications, vol. 5, no.3, pp. 357-369, 2007.

[24]Al-Akaidi M. and Daoud O., "Reducing the Peak-to-Average Power Ratio Using Turbo Coding ", IEE Proceeding Communications, vol. 153, no. 6, pp. 818-821, Dec, 2006.

[25]Yeo E., et al. "Architectures and Implementation of Low-Density Parity-Check Decoding algo ᄀrithms," in 45th IEEE Midwest Symposium on Circuits and Systems, pp. 437-440, August, 2002.

[26]Lung S.-Y., "Applied multi-wavelet feature to text independent speaker identification", IEICE Trans. Fundam. E87-A (4) 944-945, 2004. 
International Journal of Computer Networks \& Communications (IJCNC) Vol.6, No.4, July 2014

\section{Authors}

Omar Daoud (odaoud@philadelphia.edu.jo) has achieved the $\mathrm{PhD}$ in the field of Communication and Electronics Engineering at DMU/ UK 2006. He joined Philadelphia University in 2007 as Assistant Professor. His current work is about achieving the Quality of Service for the 4th Generation of the Wireless and Mobile Communication Systems by combining the advantages of the OFDM and the multiple antenna

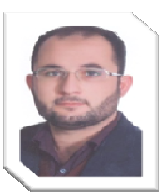
technology. He is the Assistant Dean in the Faculty of Engineering in addition to the Head of Communications and Electronics engineering department. Moreover, and in March 2012 he has promoted to the associate professor rank.

Hamarsheh Qadri received the master degree of Computer Machines, Systems and Networks from the department of Computer Engineering, Lviv Polytechnic Institute in 1991. He obtained his Ph.D degree from Lviv National University "Lvivska Polytechnica", Ukraina in 2001. Currently he is working as assistant professor of Computer Engineering, Philadelphia University-Jordan. He is in teaching since 2001. His areas of interest include Digital Signal Processing (DSP), Digital Image, Speech Processing, Object-Oriented Technology and Programming Languages, Internet Technology and Wireless Programming.

Saleh Saraireh has been an assistant Professor at Philadelphia university in Jordan Amman since 2009. I am PhD holder from the university of Sheffield, UK, in communication engineering. Also he hold a Master degree in Communication Engineering, and a Bachelor degree in electrical engineering from Mutah university, Jordan. My research area related to wireless communication, digital signal processing and cryptography.

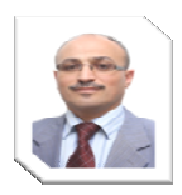

